\title{
Nucleotide sequence and analysis of the phoB-rrnE-groESL region of the Bacillus subtilis chromosome
}

\author{
Yoshito Sadaie, ${ }^{1}$ Katsunori Yata, ${ }^{1}$ Masaya Fujita, ${ }^{1}$ Hitoshi Sagai, ${ }^{2}$ \\ Mitsuhiro Itaya, ${ }^{3}$ Yasuhiro Kasahara ${ }^{4}$ and Naotake Ogasawara ${ }^{4}$
}

Author for correspondence: Yoshito Sadaie. Tel: +810559816870 . Fax: +810559816870.

e-mail: ysadaie@lab.nig.ac.jp

\footnotetext{
1 Radioisotope Center, National Institute of Genetics, Mishima 411, Japan

2 Laboratory for Pharmacology, Asahi Chemical Industry Co. Ltd, Shizuoka, Ohito 410-23, Japan

3 Mitsubishi-kasei Institute of Life Sciences, MinamiOya 11, Machida-shi, Tokyo 194, Japan

4 Advanced Institute of Science and Technology, Nara, Ikoma 630-01, Japan
}

\begin{abstract}
A $36 \mathrm{~kb}$ sequence of the phoB-rrnE-groESL region of the Bacillus subtilis chromosome at around $55^{\circ}$ has been determined. The sequenced region contains 36 ORFs including the phoB and groESL genes, and the whole rrnE operon. The phoB gene is transcribed in the direction opposite to that of chromosome replication, while most ORFs, including groESL and the $r r n E$ operon, are transcribed in the same direction. Two newly identified tRNA genes upstream of the rrnE operon were those for Arg-tRNA and Gly-tRNA. The sequenced region contains an operon consisting of genes for degradation and uptake of mannan. The rrnE operon and its downstream ORFs are well conserved among Mycoplasma genitalium, Haemophilus influenzae, Synechocystis sp. and Methanococcus jannaschii. $\sigma^{\mathrm{H}}$ consensus sequences are present in the promoter regions of three ORFs, including groESL.
\end{abstract}

Keywords: Bacillus subtilis, genome sequencing, phoB, rrnE, groESL

\section{INTRODUCTION}

Large-scale nucleotide sequencing eventually will reveal the structure and organization of genes on the chromosomes of bacteria (Bult et al., 1996; Fleischmann et al., 1995 ; Fraser et al., 1995) that have been estimated by classical genetic methods in only a limited number of organisms. A cooperative effort, by many laboratories of different countries, for sequencing the whole genome of Bacillus subtilis (Kunst et al., 1995; Ogasawara et al., 1995 ) is providing fundamental knowledge of the gene organization of the spore-forming bacterial cell. For example, sporulation of $B$. subtilis is characterized by asymmetric septation and represents one of the simplest systems of differentiation (Errington, 1994). It requires different kinds of cellular activities such as signal transduction, transcriptional regulation and protein secretion; these functions are governed by many genes on the chromosome. Knowledge of the organization of the whole genome will provide more information about genes involved in these processes.

Abbreviation: SD, Shine-Dalgarno sequence.

The GSDB, DDBJ, EMBL, NCB accession number for the nucleotide sequence reported in this paper is $D 88802$.
In this communication we describe the gene organization of the phoB-rrnE-groESL region of the B. subtilis chromosome at around $55^{\circ}$ based on the nucleotide sequence of DNA fragments derived from chromosomal fragments synthesized by long accurate PCR. This region has only a few essential genetic markers.

\section{METHODS}

DNA synthesis, random cloning and DNA sequencing. Two DNA fragments of about $15 \mathrm{~kb}$ and one fragment of $5 \mathrm{~kb}$ were synthesized by long accurate PCR (Takara LAPCR kit, Takara thermal cycler TP3000) with chromosomal DNA as template and synthetic primers. The chromosomal DNA was isolated from the cells of strain JH642 (trpC2 pheA1) (from Y. Kobayashi, Tokyo University of Agriculture and Technology, Japan) grown in $\mathrm{L}$ broth at $37^{\circ} \mathrm{C}$, by treating the cells with $1 \mathrm{mg}$ lysozyme $\mathrm{ml}^{-1}$ and $1 \%(\mathrm{w} / \mathrm{v})$ SDS in the presence of $50 \mathrm{mM}$ EDTA, followed by treatment with phenol and $20 \mu \mathrm{g}$ RNase $\mathrm{I} \mathrm{ml}^{-1}$. The sequences of the custommade primer pairs used were: 5' GGCATACAATTTTTC ACGTCTTAATGATCATCGG $3^{\prime} / 5^{\prime}$ CTCTGATAAATC CGCGGCTTTTTTCCCCCA3' ; 5'GACAGGATAGAATT CCCCACAAAGATGCCGG3' / 5'GGAACAGGCTGAGT GACTTACCTCTAAACG3 '; and 5' TGCTGTCTAAAT CCACATGG 3'/5' GACACCGCGAAGCATTGCGCG 3'. These sequences were derived from the partially determined sequences of the chromosomal NotI or Sfil linking 
Table 1. ORF analysis of the phoB-rrnE-groESL region of the $B$. subtilis chromosome

\begin{tabular}{|c|c|c|}
\hline ORF & Characteristics* & SD/initiation codon $\dagger$ \\
\hline$y d g K$ & $\begin{array}{l}\text { (nt 1-863) E. coli bicyclomycin resistance protein; P28246 (694) } \\
\text { transmembrane }\end{array}$ & \\
\hline$y d h B$ & (nt 2503-864; 259 aa) transmembrane & AAtGGAGGaaaaatatg \\
\hline$y d h C$ & $\begin{array}{l}\text { (nt } 1889-2560 ; 259 \text { aa) B. subtilis gluconate operon repressor; } \\
\text { P10585 (209) DNA binding }\end{array}$ & AAAGaggtattatatatg \\
\hline$y d h D$ & (nt 2690-4006; 439 aa) B. subtilis yaaH hypothetical protein; P37534 (704) & AAtGGgGGttaaatatg \\
\hline$y d h E$ & (nt $4155-5297 ; 381$ aa) S. lividans glycosyl transferase; JS0636 (311) & AGGCCatgtg \\
\hline$y d h F$ & (nt $6103-5342 ; 254$ aa) membrane protein & AAAGaatacaaggtg \\
\hline phoB & $\begin{array}{l}\text { (nt } 7503-6118 ; 462 \text { aa) B. subtilis alkaline phosphatase IIIA; P19405 } \\
\text { secretory (Bookstein et al., 1990) }\end{array}$ & AGGAGGT tatccagttg \\
\hline$y d h G$ & $($ nt $7829-8233 ; 135$ aa) & $\underline{\text { AGGAGGgaacaacatg }}$ \\
\hline$y d h H$ & (nt $8809-8315 ; 165$ aa) secretory & AGGAGtctttatg \\
\hline$y d h I$ & $\begin{array}{l}\text { (nt 9309-8830; } 160 \text { aa) B. subtilis yxaR hypothetical protein; } \\
\text { P46328 (512) }\end{array}$ & AGGAGtgttatg \\
\hline$y d h J$ & (nt $9392-10366 ; 325$ aa) & GAGGTGAcggcatg \\
\hline$y d h K$ & (nt $10511-11125 ; 205$ aa) membrane protein & GGgcATgttttcattg \\
\hline$y d h L$ & $\begin{array}{l}\text { (nt } 12421-11147 ; 425 \text { aa) S. lividans chloramphenicol resistance } \\
\text { protein; P31141 (492) transmernbrane }\end{array}$ & cctcaataatatggtttg \\
\hline$y d h M$ & $\begin{array}{l}\text { (nt } 12640-12948 ; 103 \text { aa) B. subtilis, cellobiose phosphotransferase system, } \\
\text { celA; P46318 (220) }\end{array}$ & $\underline{\text { AGGgGGgatagcagttg }}$ \\
\hline$y d h N$ & $\begin{array}{l}\text { (nt } 12951-13280 ; 110 \text { aa) B. subtilis cellobiose phosphotransferase } \\
\text { system celC; P46319 (233) }\end{array}$ & AAtGGtgaatcaataatg \\
\hline$y d h O$ & $\begin{array}{l}\text { (nt } 13302-14625 ; 442 \text { aa) B. subtilis cellobiose phosphotransferase } \\
\text { system celB; P46317 (998) transmembrane }\end{array}$ & GGAtagggtg \\
\hline$y d h P$ & $\begin{array}{l}\text { (nt } 14648-16042 ; 465 \text { aa) C. thermocellum } \beta \text {-glucosidase; P26208 } \\
(985)\end{array}$ & AAGGggagagagacttg \\
\hline$y d h Q$ & $\begin{array}{l}\text { (nt } 16188-16898 ; 254 \text { aa) } K \text {. aerogenes, histidine utilization } \\
\text { repressor; P12380 (199) DNA binding }\end{array}$ & GGAGGataccaatg \\
\hline$y d h R$ & (nt $16930-17826 ; 299$ aa) $S$. mutans fructokinase; Q07211 (665) & $\underline{\text { AAGGAGGCCATCtgcatg }}$ \\
\hline$y d h S$ & $\begin{array}{l}\text { (nt 17826-18770; } 315 \text { aa) B. subtilis mannose-6-phosphate } \\
\text { isomerase; P39841 (1049) }\end{array}$ & GGAGGTGcgatcatg \\
\hline$y d h T$ & $\begin{array}{l}\text { (nt } 18792-19877 ; 362 \text { aa) Bacillus sp. mannan endo-1,4- } \beta \text {-mannosidase; } \\
\text { P16699 (1049) secretory }\end{array}$ & GGAGtTGcatttg \\
\hline$y d h U$ & (nt 20396-19944; 151 aa) & AACGGgtcgtgggtg \\
\hline Arg-tRNA & (nt 21128-21204) ACG anticodon & \\
\hline $\begin{array}{l}\text { Gly-tRNA } \\
\operatorname{rrnE}\end{array}$ & (nt 21218-21291) UCC anticodon & \\
\hline $16 \mathrm{~S}$ rRNA & $($ nt $21452-23004)$ & \\
\hline $23 S$ rRNA & $($ nt 23173-26100) & \\
\hline $5 S$ rRNA & $($ nt $26156-26271)$ & \\
\hline Met-tRNA & (nt 26286-26362) CAU anticodon (Rudner et al., 1993) & \\
\hline Asp-tRNA & (nt 26423-26989) GUC anticodon (Rudner et al., 1993) & \\
\hline$y d i A$ & $\begin{array}{l}\text { (nt 26602-27654; } 351 \text { aa) H. infiuenzae hypothetical protein; E64115 (424) } \\
\text { (Rudner et al., 1993) }\end{array}$ & GGAacatcttg \\
\hline$y d i B$ & (nt 27672-28145; 158 aa) E. coli hypothetical protein; P31805 (267) & GGTGActcgtgtg \\
\hline$y d i C$ & (nt $28129-28815 ; 229$ aa) $H$. influenzae hypothetical protein ; P43990 (182) & AGGAGttaagtagacatg \\
\hline$y d i D$ & $\begin{array}{l}\text { (nt } 28828-29280 ; 151 \text { aa) } H . \text { influenzae, ribosomal protein alanine } \\
\text { acetyltransferase; } \mathrm{P} 44305(189)\end{array}$ & GGTGATaggatg \\
\hline$y d i E$ & $\begin{array}{l}\text { (nt 29276-30313; } 346 \text { aa) P. haemolytica o-sialoglycoprotein } \\
\text { endopeptidase; P36175 (660) transmembrane }\end{array}$ & GGTGACgataaatg \\
\hline$y d i F$ & $\begin{array}{l}\text { (nt } 32474-30549 ; 642 \text { aa) H. influenzae hypothetical ABC } \\
\text { transporter; P44808 (974) }\end{array}$ & GAGGTGAaataatatg \\
\hline$y d i G$ & (nt 32600-33109; 170 aa) E. coli moaC protein; P30747 (368) & AAGGAcgttt taatatg \\
\hline$y d i H$ & (nt 33109-33753; 215 aa) & GGAGGaccaaaaatg \\
\hline ydil & (nt 33778-33948; 57 aa) membrane protein & GAAAGGAGGagcccaaatatg \\
\hline
\end{tabular}


Table 1. Cont.

\begin{tabular}{|c|c|c|}
\hline ORF & Characteristics* & $\mathrm{SD} /$ initiation codon $\uparrow$ \\
\hline$y d i]$ & $\begin{array}{l}\text { (nt } 33958-34719 ; 254 \text { aa) B. subtilis } y c b T \text { hypothetical protein } \\
\text { P42252 (519) transmembrane }\end{array}$ & $\underline{\text { AGAAAGaagatcagtaagttaggatg }}$ \\
\hline$y d i K$ & (nt $34951-34763 ; 63$ aa) secretory & GGAGGattatttttatg \\
\hline$y d i L$ & (nt 35682-34951; 244 aa) transmembrane & AAAGGAGtacataccatttg \\
\hline groES & (nt 35879-36202; 108 aa) (Schmidt et al., 1992; Tozawa et al., 1992) & ctcttagtgctgagtg \\
\hline groEL & (nt 36252-36308) (Schmidt et al., 1992; Tozawa et al., 1992) & $\underline{\text { GGAGGtcttgtaaacatg }}$ \\
\hline
\end{tabular}

* The characteristics of the ORFs comprise: (the beginning and end nucleotide numbers and the size of the protein in aa); the homologous protein and its accession number (SWISS-PROT); the FASTA score in parentheses [ $\geqslant 182$; by DDBJ Homology Search System (see Methods $)$; protein features [by PSORT Prediction Program (see Methods)]; and reference.

†SD sequence in underlined upper case and initiation codon in bold.

fragments in pNEXT26 and pSOFT8 (Itaya \& Tanaka, 1991), a $\lambda$ DASHII clone (from the B. subtilis DNA library of J. Nakai, University of Osaka, Japan) located between these linking genes and from the sequence of groESL (Schmidt et al., 1992; Tozawa et al., 1992).

The resulting DNA fragments of about $15 \mathrm{~kb}$ and $5 \mathrm{~kb}$ were purified by agarose gel electrophoresis, partially digested with DNase I (Takara), and cloned into the M13mp19 (Takara) cloning vector as described elsewhere (Yoshida et al., 1995). A sample of DNA $(20 \mu \mathrm{g})$ was digested with $0.0056 \mathrm{U}$ DNase I at $37^{\circ} \mathrm{C}$ for $4.5 \mathrm{~min}$ in $400 \mu \mathrm{l}$ reaction mixture $(50 \mathrm{mM}$ Tris $/ \mathrm{HCl}$ $\mathrm{pH} 7 \cdot 5,10 \mathrm{mM} \mathrm{MnCl}, 100 \mu \mathrm{g} \mathrm{BSA} \mathrm{ml}^{-1}$ ). PCR screening was used to select for clones with inserts of sufficient size ( $>400 \mathrm{bp}$ ). These clones were selected randomly and subsequently sequenced. Some regions were difficult to clone into phage vectors. Two of these regions, one of them located between $y d h L$ and $y d h M$ and the other between $y d b U$ and the $16 S$ rRNA genes, were directly sequenced with DNAs synthesized with primers outside the regions. Several primers were prepared and used to sequence the DNA covering the gap region.

Sequencing was performed with a DNA sequencer (373A, Applied Biosystems) using a Taq Dye Primer and Dye Terminator Cycle Sequencing Kit (Applied Biosystems). Sequences obtained were assembled into contigs using a personal computer with the AutoAssembler (Version 1.3, Applied Biosystems).

Computer analysis. The DNA sequences determined were analysed with GeneWorks (version 2.0, IntelliGenetics), DISC Homology Search (http://www.dna.affrc.go.jp/htbin/ prefasta), PSORT Prediction (http://psort.nibb.ac.jp/ form.html), DDBJ Homology Search System (http:// crick.genes.nig.ac.jp:8080/fasta_blast/html/ Welcome.html), OWL (http://www.biochem.ucl.ac.uk/ bsm/dbbrowser/OWL/OWL.html), TIGER'S WWW Server (http://www.tigr.org/) and CyanoBase (http:// www.kazusa.or.jp/cyano/cyano.html).

\section{RESULTS AND DISCUSSION} DNA sequence of the phoB-rrnE-groESL region of
the B. subtilis $\mathrm{JH} 642$ chromosome at around $55^{\circ}$

We first partially sequenced two DNA fragments, pNEXT26 and pSOFT8, described as linking clones

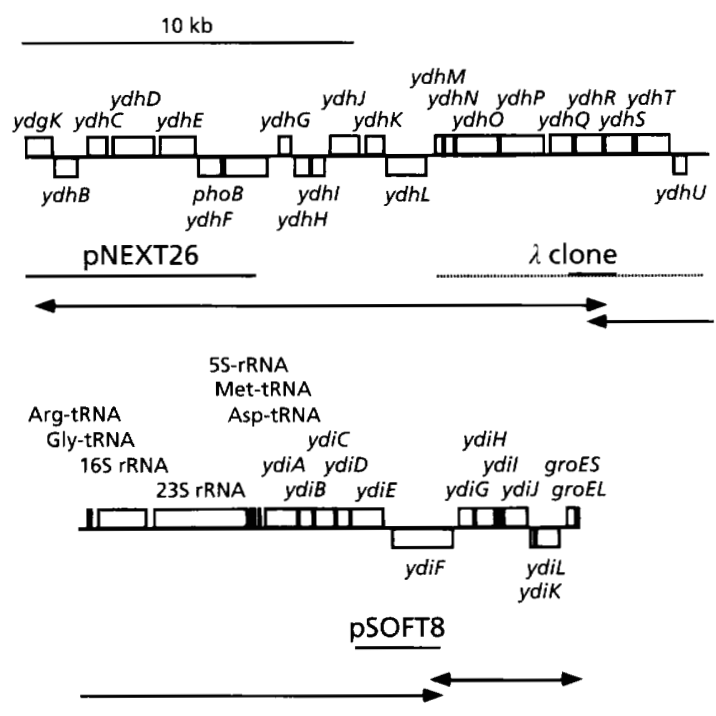

Fig. 1. ORFs and RNA genes in the phoB-rrnE-groESL region of the $B$. subtilis JH642 chromosome. Transcription is from left to right (upper boxes) and right to left (lower boxes). Direction of chromosome replication is from left to right. Thick bars indicate Notl and Sfil linking fragments in pNEXT26 and pSOFT8. The 2DASHII clone used for the partial sequencing is also indicated. Arrows indicate DNA fragments synthesized by PCR.

(Itaya \& Tanaka, 1991) by the deletion method and the region between the above clones which was obtained as a hybridizable $\lambda$ DASHII clone. Based on the results obtained, we synthesized, by long accurate PCR, two DNA fragments each about $15 \mathrm{~kb}$ long which covered the above region, and one short DNA fragment of $5 \mathrm{~kb}$ long covering the region between pSOFT8 and groESL, and sequenced shorter fragments derived from these three DNAs after random digestion with DNase I and subcloning in M13mp19.

In total, $36 \mathrm{~kb}$ of DNA was sequenced (Fig. 1). All parts were sequenced on both strands, except for some regions in the $r r n E$ and groESL genes where only one strand was 


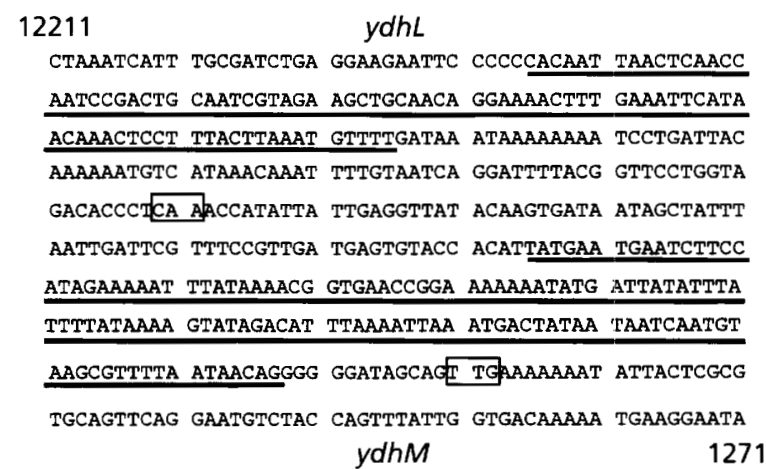

Fig. 2. DNA sequence of the promoter region of $y d h L$ and $y d h M$. Underlined regions indicate DNA sequences determined from only one strand. Start codons are boxed.

sequenced and compared with those sequences already described (Rudner et al., 1993; Schmidt et al., 1992; Tozawa et al., 1992). The region between the $y d b L$ and $y d h M$ genes, which are transcribed in opposite directions, could not be sequenced on both strands. The sequences, nt 12245-12335 and nt 12495-12627 could be determined from only one strand. The former sequence was obtained from the DNA template complementary to the sequence shown in Fig. 2, and the latter sequence from a template DNA, the sequence of which is shown in Fig. 2. Sequencing from the opposite strand resulted in the frequent occurrence of $\mathrm{N}$ (any of the four possible nucleotides), probably because of non-specific or fairly equal incorporation of ddNTP. DNAs showing an Nrich pattern have sequences that are the same as those of the $5^{\prime}$ portion of the presumed messenger RNAs of the $y d h L$ and $y d h M$ genes. Template DNA may take on tertiary structures and cause $\mathrm{N}$-rich sequences by blocking DNA synthesis at specific points.

\section{ORF analysis of the sequenced region}

ORFs beginning with an ATG, GTG or TTG start codon and comprising more than 100 codons, were searched. As shown in Fig. 1 and Table 1, there were 36 ORFs, including the phoB (Bookstein et al., 1990) and groES/groEL (Schmidt et al., 1992; Tozawa et al., 1992) genes. Except for $y d h L$ and groES, Shine-Dalgarno (SD) sequences were detectable in these genes. The SD sequence is the complementary sequence to the $3^{\prime}$ end sequence (UCUUUCCUCCACUAG...) of the $16 \mathrm{~S}$ rRNA of B. subtilis. Twenty one, eight and six ORFs, respectively, had an ATG, TTG or GTG start codon.

The $y d h B$ gene encodes a putative transmembrane protein with unknown function and has a $\sigma^{\mathrm{H}}$ promoter consensus sequence AGGAN 16 GAAT (Haldenwang, 1995).

The $y d h C, y d h D$ and $y d h E$ genes seem to form an operon whose controlling element might be the $y d h C$ gene as it encodes a putative protein with a DNA
HTH Motif

YdhC

GntR

YdhQ

HutC

. . GERINETQLAKS FG VSRSPIREAMRLLEKD . . . . GTILSENTIAAEFS VSRSPVREALKILASE . .

. QP IPDEVS IAKE FNSSRMTMKRALDNLVAE. . . .DR IPS EAE IVAOFGF SRMTINRALRELTDE. .

Fig. 3. Comparison of helix-turn-helix (HTH) motifs of YdhC and YdhQ with those of GntR (B. subtilis gluconate operon repressor) and HutC (Klebsiella aerogenes histidine utilization repressor). HTH regions are underlined. Amino acids common to all sequences are shown in bold letters. Identical amino acids between YdhC and GntR, and between YdhQ and HutC are indicated by italic letters.

binding domain similar to that of the gluconate operon repressor (Fujita et al., 1986) (Fig. 3).

The $y d h F$ gene, which encodes a transmembrane protein, may form an operon with the alkaline phosphatase gene.

The $y d h H$ gene presumably encodes a secretory protein with a signal sequence. $y d h I$ has a homologue $(y x a R)$ on the B. subtilis chromosome.

The region from $y d h M$ to $y d h T$ may constitute a large operon involved in degradation and incorporation of mannan, although we need the transcription analysis to determine whether or not these genes really constitute a single operon. This hypothesis is based on the observation that the first three ORFs ( $y d h M, y d h N, y d h O)$ encode proteins homologous to the proteins involved in the phosphotransferase system of sugar uptake (PTS), where $y d b O$ encodes a transmembrane protein. Following this putative operon, four ORFs encoding proteins with high similarity to sugar assimilation proteins are present: glucosidase $(y d h P)$, fructokinase $(y d h R)$, mannose-6-phosphate isomerase $(y d h S)$ and mannanase $(y d h T)$. YdhT has a putative signal sequence; hence it is likely to be a secreted protein. This operon might have an internal repressor as $y d h Q$ encodes a protein having a DNA binding domain similar to that of the histidine utilization operon repressor, HutC (Schwacha \& Bender, 1990) (Fig. 3).

The region between $y d b U$ and $r r n E$ has no ORFs (having more than 30 codons) with similarity to any known protein.

Five ORFs from $y d i A$ (Rudner et al., 1993) to $y d i E$, may constitute a large operon involved in macromolecule degradation as the last of these ORFs ( $y d i E$ ) encodes a protein homologous to the glycoprotease.

The $y d i K$ and $y d i L$ genes probably constitute an operon. YdiL is a transmembrane protein and YdiK has a signal sequence and hence must be secreted. $y d i K$ has a $\sigma^{\mathrm{H}}$ consensus promotor sequence, AGGAN ${ }_{15}$ GAAT (Haldenwang, 1995).

groES has a $\sigma^{\mathrm{H}}$ consensus promotor sequence,

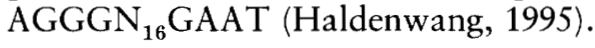




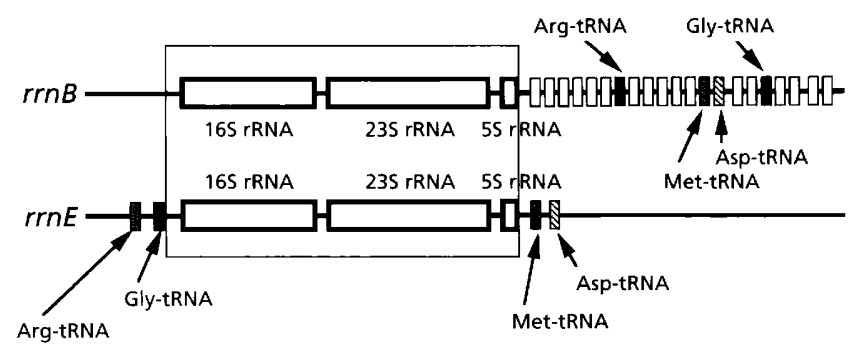

Fig. 4. The $r r n B$ and $r r n E$ regions of the $B$. subtilis chromosome.

\section{The rrnE region}

The $r r n E$ region consists of four tRNA genes and three rRNA genes. We compared the sequence of the $r r n E$ region and $r r n B$ region as the latter also carries a cluster of tRNA genes (Green et al., 1985). In the $r r n E$ region, two tRNAs, for Arg and Gly, precede the consecutive $16 \mathrm{~S}, 23 \mathrm{~S}$ and $5 \mathrm{~S}$ rRNA genes, which are followed by two tRNA genes for Met and Asp (Rudner et al., 1993) (Fig. 4). These four tRNA genes are all found in the tRNA cluster of the $r r n B$ region, although in the latter case these genes are all located behind the rRNA genes.

The second tRNA gene of the rrnE region, Gly-tRNA has two mutations (nt 21267, A; nt 21277, T) compared to Gly-tRNA (G, C) of the $r \mathrm{rnB}$ operon. These mutations occur pairwise at two sites of the T $\Psi \mathrm{C}$ stem of tRNA. The other three tRNA genes of the $r r n E$ region are identical to those of the $r m B$ operon.

DNA sequences of $r r n E$ and $r r n B$ are well conserved with only one or two mutations over 100 bases from the end of Gly-tRNA to the end of $5 \mathrm{~S}$ rRNA, including the spacer regions (boxed in Fig. 4).

\section{Genes conserved among five bacterial species}

B. subtilis genome sequences were compared with those from four other bacterial species: Synechocystis sp., Haemophilus influenzae, Methanococcus jannaschii and Mycoplasma genitalium. Similarity searches of our ORFs and RNA genes with those of the other bacteria gave the results shown in Table 2, where $\operatorname{rrnE}(16 \mathrm{~S}$ and 23S) and the genes located downstream of $r r n E$ (especially $y d i E$, a glycoprotein protease gene) are well conserved. Whereas groEL is also well conserved, groES is not. The genes of this region may have been conserved during evolution although the gene organization seems not to be conserved.

\section{ACKNOWLEDGEMENTS}

We thank Roy H. Doi for critical reading of the manuscript and helpful comments. This work was supported by a Grantin-Aid for Creative Basic Research on 'Human Genome Analysis' from the Ministry of Education, Science and Culture of Japan.

\section{Table 2. Genes conserved among five bacterial species}

Homology searches were carried out using the Tiger Database GRASTA program

(http: //www.tigr.org/tdb/mdb/hidb/hidb.html, http: //www.tigr.org/tdb/mdb/mjdb/mjdb.html and http ://www.tigr.org/tdb/mdb/mgdb/mgdb.html) and the CyanoBase BLASTP program

(http://www.kazusa.or.jp/cyano/cyano.html). Scores ( $>100)$ indicate B. subtilis (Bs) homologues in Haemophilus influenzae (Hi), Methanococcus jannaschii (Mj), Mycoplasma genitalium (Mg) and Synechocystis sp. (Ss).

\begin{tabular}{|c|c|c|c|c|}
\hline \multirow[t]{2}{*}{ Bs } & \multicolumn{4}{|c|}{ Homology scores } \\
\hline & $\mathrm{Hi}$ & $\mathbf{M j}$ & $\mathrm{Mg}$ & Ss \\
\hline$y d g K$ & 677 & & & 114 \\
\hline$y d h B$ & 127 & & & 140 \\
\hline$y d b C$ & 106 & & & \\
\hline$y d b D$ & & & & \\
\hline$y d h E$ & & & & 180 \\
\hline$y d h F$ & & & & \\
\hline phoB & & & & \\
\hline$y d h G$ & & & & \\
\hline$y d h H$ & & & & \\
\hline$y d h I$ & & & & \\
\hline$y d h J$ & & 204 & 136 & \\
\hline$y d b K$ & & & & \\
\hline$y d h L$ & 406 & 137 & 106 & 132 \\
\hline$y d h M$ & & & & \\
\hline$y d h N$ & & & & \\
\hline$y d b O$ & & 120 & & \\
\hline$y d h P$ & & & & \\
\hline$y d h Q$ & & & & \\
\hline$y d h R$ & & & & 103 \\
\hline$y d h S$ & & & & \\
\hline$y d h T$ & & & & \\
\hline$y d h U$ & & & & \\
\hline Arg-tRNA & 232 & 151 & 268 & \\
\hline Gly-tRNA & 191 & & 244 & 274 \\
\hline $16 \mathrm{~S}$ rRNA & 4329 & 2197 & 3918 & 2539 \\
\hline 235 rRNA & 7078 & 3527 & 7139 & 2763 \\
\hline $5 S$ rRNA & 331 & 196 & 150 & \\
\hline Met-tRNA & 340 & 226 & 268 & 146 \\
\hline Asp-tRNA & 306 & & 115 & 127 \\
\hline$y d i A$ & 388 & 173 & & 164 \\
\hline$y d i B$ & 232 & & & 188 \\
\hline$y d i C$ & 172 & & & 111 \\
\hline$y d i D$ & 175 & 138 & & 161 \\
\hline$y d i E$ & 686 & 353 & 438 & 295 \\
\hline$y d i F$ & 911 & 181 & 135 & 669 \\
\hline$y d i G$ & 365 & 235 & & 147 \\
\hline$y d i H$ & & & & \\
\hline$y d i I$ & & & & 114 \\
\hline$y d i J$ & & & & 135 \\
\hline$y d i K$ & & & & \\
\hline$y d i L$ & & & & 108 \\
\hline groES & 212 & & & 266 \\
\hline groEL & 1430 & 245 & 1094 & 633 \\
\hline
\end{tabular}




\section{REFERENCES}

Bookstein, C., Edwards, C. W., Kapp, N. V. \& Hulett, F. M. (1990). The Bacillus subtilis 168 alkaline phosphatase 111 gene; impact of a phoAIII mutation on total alkaline phosphatase synthesis. J Bacteriol 172, 3730-3737.

Bult, C. J. and others (1996). Complete genome sequence of the methanogenic Archaeon, Methanococcus jannascbii. Science 273, 1058-1073.

Errington, J. (1994). Bacillus subtilis sporulation; regulation of gene expression and control of morphogenesis. Microbiol Rev 57, $1-33$.

Fleischmann, R. D. and others (1995). Whole-genome random sequencing and assembly of Haemophilus influenzae Rd. Science 269, 496-512.

Fraser, C. M. and others (1995). The minimal gene complement of Mycoplasma genitalium. Science 270, 397-404.

Fujita, Y., Fujita, T., Miwa, Y., Nihashi, J. \& Aratani, Y. (1986). Organization and transcription of the gluconate operon, gnt, of Bacillus subtilis. J Biol Chem 261, 13744-13753.

Green, C. J., Stewart, G. C., Hollis, M. A., Vold, B. S. \& Bott, K. F. (1985). Nucleotide sequence of the Bacillus subtilis ribosomal RNA operon, rrnB. Gene 37, 261-266.

Haldenwang, W. G. (1995). The sigma factors of Eacillus subtilis. Microbiol Rev 59, 1-30.

Itaya, M. \& Tanaka, T. (1991). Complete physical map of the Bacillus subtilis 168 chromosome constructed by a gene-directed mutagenesis method. J Mol Biol 220, 631-648.

Kunst, F., Vassarotti, A. \& Danchin, A. (1995). Organization of the European Bacillus subtilis genome sequencing project. Microbiology 141, 249-255.
Ogasawara, N., Nakai, S. \& Yoshikawa, H. (1994). Systematic sequencing of the 180 kilobase region of the Bacillus subtilis chromosome containing the replication origin. DNA Res 1, 1-14.

Ogasawara, N., Fujita, Y., Kobayashi, Y., Sadaie, Y., Tanaka, T., Takahashi, H., Yamane, K. \& Yoshikawa, H. (1995). Systematic sequencing of the Bacillus subtilis genome: progress report of the Japanese group. Microbiology 141, 257-259.

Rudner, R., Chevrestt, A., Bucholz, S. R., Studamire, B., White, A. M. \& Jarvis, E. D. (1993). Two tRNA gene clusters associated with rRNA operons $r r n D$ and $r r n E$ in Bacillus subtilis. $J$ Bacteriol 175, 503-509.

Schmidt, A., Schiesswohl, M., Voelker, U., Hecker, M. \& Schumann, W. (1992). Cloning, sequencing, mapping and transcriptional analysis of the groESL operon from Bacillus subtilis. J Bacteriol 174, 3993-3999.

Schwacha, A. \& Bender, R. A. (1990). Nucleotide sequence of the gene encoding the repressor for the histidine utilization genes of Klebsiella aerogenes. J Bacteriol 172, 5477-5481.

Tozawa, Y. M., Itaya, M., Yoshikawa, H., Kawamura, F. \& Takahashi, H. (1992). Isolation and characterization of the groES/groEL operon of Bacillus subtilis. Biosci Biotech Biochem 56, 1995-2002.

Yoshida, K., Sano, H., Seki, S., Oda, M., Fujimura, M. \& Fujita, Y. (1995). Cloning and sequencing of a $29 \mathrm{~kb}$ region of the Bacillus subtilis genome containing the hut and wapA loci. Microbiology 141, 337-343.

Received 11 November 1996; revised 14 January 1997; accepted 17 January 1997 